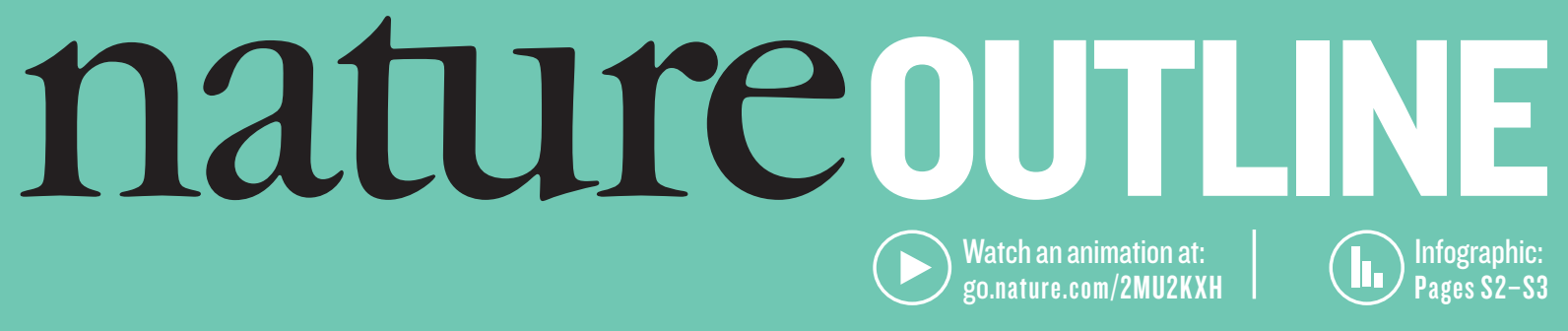

\title{
RETINAL REPAIR: VISIONS OF THE FUTURE
}

\author{
Damage to the retina has so far proved \\ irreversible, but stem-cell therapies \\ could hold the key to restoring sight.
}

\section{BY DAVID HOLMES}

r or an idea of how difficult life can be for people with advanced age-related macular degeneration (AMD), focus your eyes farther up the page and try to reread this sentence using just your peripheral vision. People with advanced AMD see a severely distorted image or a large black spot at the centre of their vision, making everyday tasks such as reading, cooking or driving extremely frustrating, if not impossible. With no cure available, those affected will carry the condition for the rest of their lives.

$\mathrm{AMD}$ is the most common of a group of eye diseases caused by the degeneration of the retina - the thin layer of tissue at the back of the eye that transforms light into electrical signals that are then translated into an image by the brain. Around 196 million people worldwide will develop AMD by 2020. Several million others will be affected by less common, hereditary forms of retinal degeneration such as retinitis pigmentosa, a group of disorders that first affects the peripheral vision, and often develops in early childhood.

Given the scale of the problem, it is no surprise that researchers are working to better understand the causes of retinal degeneration, as well as exploring various therapeutic approaches in the hope of restoring lost vision.

Leading the pack are several potential treatments based on stem cells, which have the capacity to take on the form of any cell type that contributes to the retina's intricate structure. Already, several procedures that involve the implantation of retinal cells derived from stem cells have reached trials in people. In March, the London Project to Cure Blindness - a collaboration between researchers at University College London and Moorfields Eye Hospital - announced the results of a trial in which two people with AMD received a bioengineered patch containing retinal cells produced from human embryonic stem cells (L. da Cruz et al. Nature Biotechnol. 36, 328-337; 2018).

The patch was made by coaxing stem cells to differentiate into retinal pigment epithelium - a monolayer of cells that forms the interface between the retina and the circulatory system, and that is damaged in people with AMD. Clinicians were then able to replace a section of damaged epithelium with healthy cells by surgically delivering the patch to the base of the retina. Both

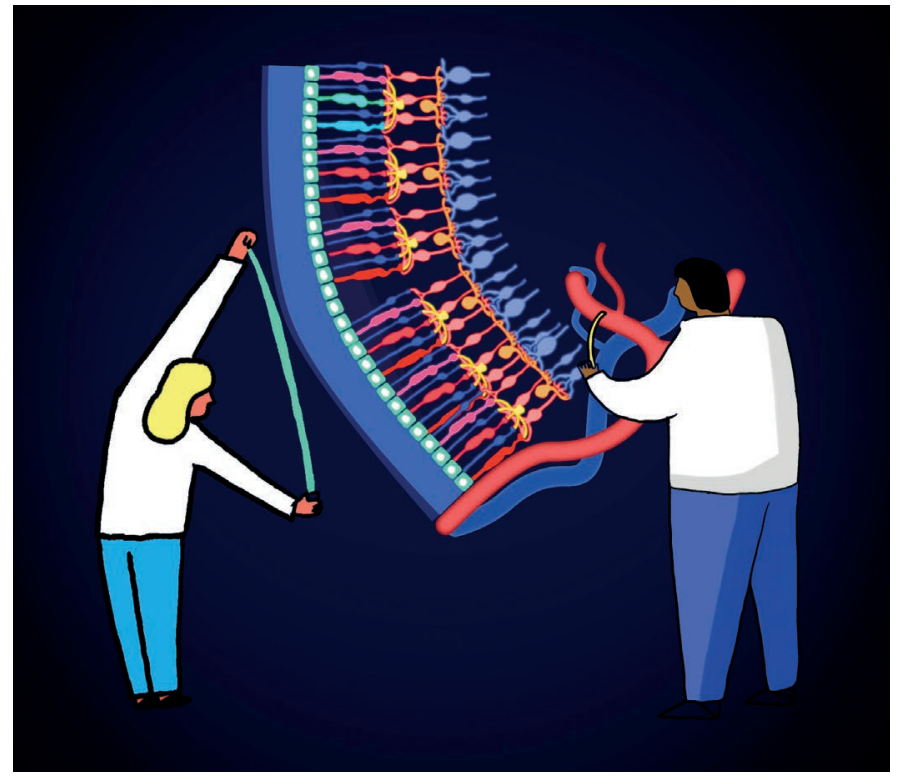

recipients tolerated the procedure well, echoing results from a similar trial in Japan (M. Mandai et al. N. Engl. J. Med. 376, 1038-1046; 2017). However, unlike those in the Japanese study, both participants in the London Project's study reported an improvement in their vision, with one going from being almost unable to read to reading more than 80 words per minute.

Although these trials are too small to draw firm conclusions about whether stem-cell therapies will provide a cure for AMD, these promising findings have energized the field. And as stem-cell culturing techniques continue to be refined, the prospect of growing not only individual cell types but whole, functional retinas moves closer. Such systems could become a powerful tool for drug discovery and disease modelling. Researchers have already created retinal organoids that bear a striking similarity to human retinas, in terms of cell composition and organization. For people affected by retinal degeneration, the future is starting to look much clearer.

Nature is pleased to acknowledge the financial support of the NIHR Biomedical Research Centre at Moorfields Eye Hospital NHS Foundation Trust and UCL Institute of Ophthalmology. As always, Nature retains sole responsibility for all editorial content.

David Holmes is a science writer in the United Kingdom. Health Research
Nature Outlines are sponsored supplements that aim to unpack scientific and technical concepts through infographics, illustrations and short animations. The boundaries of sponsor involvement are clearly delineated in the guidelines available at http://go.nature.com/ecx76b. Feedback@nature.com.Copyright $($ C 2018 Springer Nature Limited.All rights reserved 